\title{
An efficient method for indexing grazing-incidence X-ray diffraction data of epitaxially grown thin films
}

\author{
J. Simbrunner', B. Schrode ${ }^{2}$, J. Domke ${ }^{3}$, T. Fritz ${ }^{3}$, R. Forker ${ }^{3}$, R. Resel ${ }^{2}$ \\ ${ }^{1}$ Division of Neuroradiology, Vascular and Interventional Radiology, Medical University Graz, Austria, \\ ${ }^{2}$ Institute of Solid State Physics, Graz University of Technology, Austria, \\ ${ }^{3}$ Institute of Solid State Physics, Friedrich Schiller University Jena, Germany
}

josef.simbrunner@medunigraz.at

In case of grazing incidence X-ray diffraction (GIXD), as usually performed on fibre textured films, only two components (of the total three) of the reciprocal lattice vectors - namely an out-of plane component $\mathrm{q}_{\mathrm{z}}$ and an in-plane component $\mathrm{q}_{\mathrm{xy}}$ - are available for the indexing procedure. In previous work, we have presented an algorithm for indexing such diffraction patterns, where the additional presence of a specular diffraction peak is being explicitly taken into account [1]. In the present work, we now aim to formulate this indexing method for a number of GIXD patterns collected for samples at different azimuthal rotation angles account [2]. Thus, all three components of the scattering vector are obtained. Also in this case, the combination of the diffraction peaks obtained from GIXD with the specular diffraction peak(s) simplifies the indexation procedure, so that finally, different phases of epitaxially oriented films can be identified.

In theory, the parameters of the reduced unit cell and its orientation can simply be obtained from the matrix of three linearly independent reciprocal space vectors. However, if the sample exhibits unit cells in various alignements and/or with different lattice parameters, it is necessary to assign all experimentally obtained reflections to their associated individual origin. An effective algorithm is described to accomplish this task in order to determine the unit-cell parameters of low symmetry systems comprising different orientations and polymorphs. Our method is particularly advantageous if the number of reflections is relatively small or the sample consists of various crystal lattices or alignments, as it is commonly found for organic thin films grown on single crystalline substrates. For easy access to epitaxial relationships, the lattice constants of the involved unit cells and the parameters of the orientation matrix can be determined simultaneously.

Well-known (PTCDA, pentacene), as well as crystallographically less characterized samples (trans-DBPen, DCV4T-Et2) on various substrates were analyzed [3]. In all cases, crystallographic unit cells exhibiting specific contact planes with the substrate were obtained. Additionally, distinct $60^{\circ}$ symmetries for the positive and negative orientations of the contact plane were found. In the particular case of DCV4T-Et2 grown on $\mathrm{Ag}(111)$, three new polymorphs with different contact planes and cell parameters were found (see Figure 1); when using graphene/ $\mathrm{SiC}(0001)$ as substrate, however, only one of these polymorphs could be observed. Our work shows that indexing is possible even when different alignments of crystals occur within a thin film and also in the presence of several polymorphs.
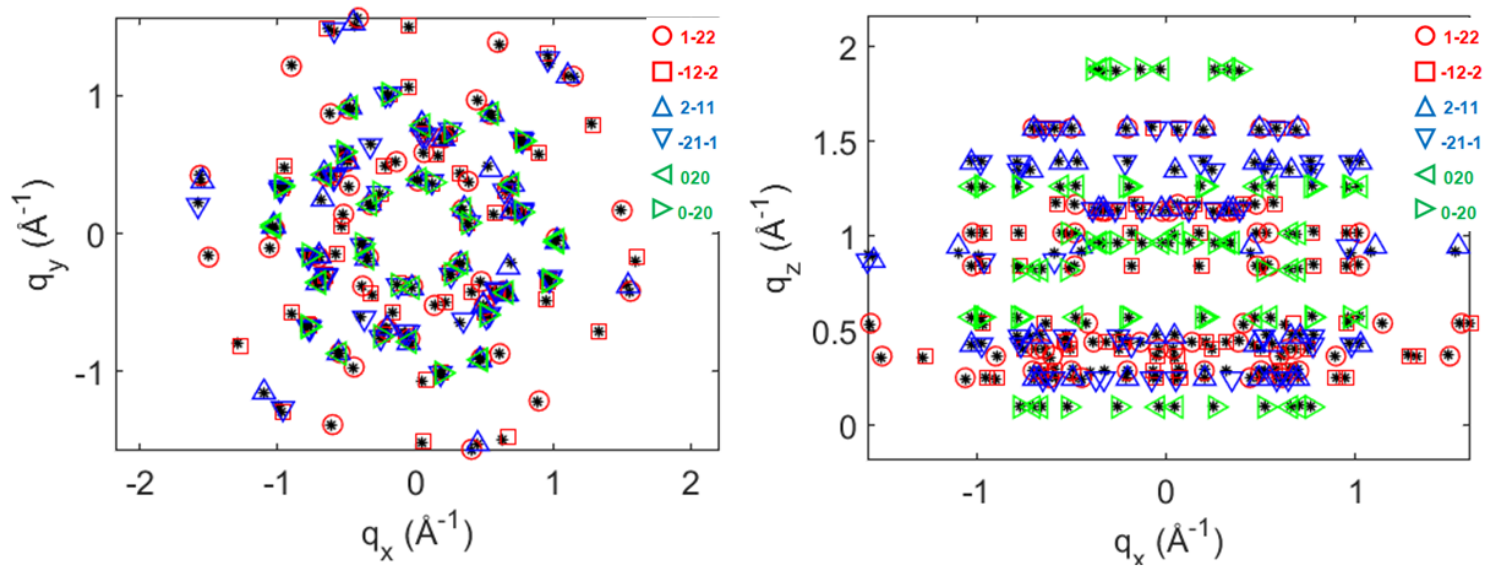

Figure 1. Positions of experimentally determined X-ray diffraction peaks (black) of DCV4T-Et2 crystals grown on Ag(111, obtained from rotating GIXD experiments. $q_{y} / q_{x}$ position of the diffraction peaks (left); $q_{z} / q_{x}$ positions of the diffraction peaks (right). Indexing

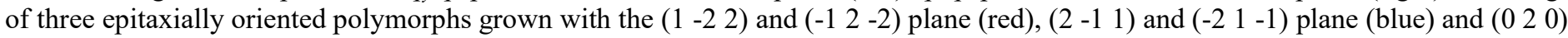
and $(0-20)$ plane (green), respectively parallel to the substrate surface. 


\section{PS-54-5}

\section{Poster Session}

[1] Simbrunner, J., Simbrunner, C., Schrode, B., Röthel, C., Bedoya-Martinez, N., Salzmann, I. \& Resel, R. (2018). Acta Cryst. A74, 373.

[2] Simbrunner, J., Schrode, B., Domke, J., Fritz, T, Salzmann, I. \& Resel, R. (2020). Acta Cryst. A76, 345.

[3] Simbrunner, J., Schrode, B., Hofer, S., Domke, J., Fritz, T, Forker, R. \& Resel,, R. (2021). J. Phys. Chem. C125: 618.

Keywords: organic epitaxy; GIXD; indexing; polymorphism 